\title{
Correlation Between Anosmia and Severity Along with Requirement of Tocilizumab in COVID-19 Patients
}

\author{
Manju Silu ${ }^{1}$ (D) Navneet Prasad Mathur ${ }^{1} \cdot$ Rekha Kumari $^{1} \cdot$ Pallavi Chaudhary $^{1}$
}

Received: 3 April 2021 / Accepted: 7 June 2021/Published online: 17 June 2021

(C) Association of Otolaryngologists of India 2021

\begin{abstract}
Anosmia with or without dysgeusia is frequently associated with Severe acute respiratory syndrome coronavirus 2 (SARS-CoV-2) SARS-CoV-2 infection. SARS$\mathrm{CoV}-2$ virus affects the olfactory system and thus represents neurotropic and neuro-invasive nature of the virus. We found that tocilizumab's role in reducing mortality in severe covid-19 infection is still questionable and aim of our study was correlation of anosmia and severity of covid19 infection and requirement of tocilizumab in anosmia patients. To establish relationship between anosmia and severity of COVID-19 infection along with requirement of tocilizumab. This was a retrospective cum prospective cross sectional study done on COVID-19 patients who were admitted in normal COVID-19 ward and intensive care unit of Employee's State Insurance Corporation Hospital, Udaipur and Maharaja Bhupal Hospital of Ravindra Nath Medical College between September 2020 and 15 January 2021 age group of 18 to 92 years, both sexes. We had asked about anosmia in all COVID-19 positive patients and followed all patients in perspective of severity of disease and tocilizumab. $p$ value $<0.001$ of prevalence of anosmia in COVID -19 patients who were isolated at home, admitted in ward and intensive care unit
\end{abstract}

Manju Silu

manjusilu35@gmail.com

Navneet Prasad Mathur

navneetmathur12@yahoo.co.in

Rekha Kumari

rekhachoudhary0507@gmail.com

Pallavi Chaudhary

trinabhandpallavi@gmail.com

1 Department of Otorhinolaryngology, Ravindra Nath Medical College, Udaipur, Rajasthan, India showed that anosmia is inversely related to severity of disease. Tocilizumab has no significant role in decreasing mortality in severe form of disease. Our study indicates that anosmia is related to the mildness of disease and there is no role of tocilizumab in decreasing the mortality in severe form of disease.

Keywords Anosmia - COVID-19 · Tocilizumab . Severity · Wuhan

\section{Introduction}

Severe acute respiratory syndrome coronavirus 2 (SARSCoV-2) is liable for COVID-19 or coronavirus pandemic which is an ongoing pandemic of coronavirus disease 2019 which was started first in December 2019, in Wuhan city of Hubei province (China) thereafter rapidly spread in Europe and Worldwide. First case of COVID -19 in India was reported on 30 January and now India has highest number of confirmed cases in Asia and third in world after United States and Brazil (as per WHO report till 25march). Fatality rate of covid-19 in India is $1.45 \%$ (As per data provided by ministry of Health). In COVID-19 infection, presentation of patient's clinical symptoms is diverse. Patient can be asymptomatic or severe pneumonia with respiratory distress that requires mechanical ventilation or severe pneumonia that leads to death [1-3]. Anosmia with or without dysgeusia is frequently associated with SARSCoV-2 infection. In general change in odor is due to inflammatory responses against viral infection, but COVID-19 related anosmia is present without conventional inflammatory or coryzal symptoms which are generally absent in COVID-19 patients [4-7]. SARS-CoV-2 virus affects the olfactory system and thus represents neurotropic 
and neuro-invasive nature of the virus [8-12]. Once the virus gets entry to human body it replicates during initial phase and may or may not followed by inflammatory response by the host in later phase [13]. In severe disease SARS-CoV-2 infection causes hyper immune response which leads to acute respiratory distress syndrome and there is cytokine storm in most critical patients which is characterized by long term damage and lung fibrosis due to increased production of variety of cytokines [14, 15]. At present management of COVID-19 pneumonia is approached with combination of antiviral and immunomodulatory drugs including tocilizumab, a recombinant humanized monoclonal antibody of the IgG1 class against the interleukin-6 receptor. Both forms (soluble and membrane bound) of IL-6 are targeted by tocilizumab [16, 17]. After thorough search on PubMed, Embase, Cochrane Review and other online libraries we found that tocilizumab's role in reducing mortality in severe COVID-19 infection is still questionable and aim of our study to ascertain the role of anosmia in risk stratification and predicting COVID-19 patients' prognosis.

\section{Aims and Objectives}

To establish relationship between anosmia and severity of COVID-19 infection along with requirement of tocilizumab.

\section{Material and Methods}

This was a retrospective cum prospective cross sectional study done on COVID-19 patients (confirmed by Real Time-Polymerase Chain Reaction on nasopharyngeal swab $\{\mathrm{RTPCR}\}$ ) who were isolated at home, admitted in COVID-19 ward and intensive care unit (ICU) of Employee's State Insurance Corporation Hospital, Udaipur and Maharaja Bhupal Hospital of Ravindra Nath Tagore (RNT) Medical College, Udaipur between September 2020 and 15 January 2021 age group of 18 to 92 years, both sexes. The study was approved by the Ethical Committee of Ravindra Nath Medical College, Udaipur. Verbal informed consent was taken from all patients due to safety and isolation precautions. We included all RTPCR confirmed COVID -19 patients, age above 18 years and give verbal consent to participate in the study. Patients who didn't give consent, lost follow up (not responded on telephonic call) and no record about anosmia in records (for mechanically ventilated ICU patients) were excluded from the study. We had asked about anosmia in all COVID-19 positive patients and follow up of all patients in perspective of severity of disease and who received tocilizumab. In our hospital we admitted only those patients who developed shortness of breath and other clinical features of moderate to severe COVID-19 disease and rest of patients were isolated at home for 28 days. Patient who had anosmia but no breathlessness were isolated at home and we had followed all patients via telephonic call about their illness until 42 days. COVID-19 anosmia reporting tool developed by department of Otorhinolaryngology and Head and Neck Surgery, RNT Medical College, Udaipur was used for anosmia of home isolated and ward patients. Data were collected from admission records of ICU patients. We simply asked to patients, whether they have anosmia or not and for severity of anosmia we didn't follow any questionnaire. At the end of 42 days our outcome tool includes:

1. Whether patient needed admission or not(for home isolation patients)

2. Whether patient discharged or shifted to ICU (for Covid-19 ward patients)

3. Whether patient progress to need of mechanical ventilation or shifted to COVID-19 wards(for those ICU patients who were not mechanically ventilated)

4. Whether patient survived or not (for mechanically ventilated ICU patients)

\section{COVID-19 Anosmia Reporting Tool}

Department of Otorhinolaryngology and Head and Neck Surgery Ravindra Nath Tagore Medical College, Udaipur.

1. Data provided by any medical specialist or patient him or herself

2. Patient age

3. Patient gender

4. Is the source of the COVID-19 infection identifiable? Yes or No

5. Other Risk factors

None, smoking, head trauma, Sinusitis/ allergy, Chronic respiratory disease/Asthma, Cardiac disease, Neurologic disease (e.g. Parkinson's), other

6. When was the anosmia (loss of sense of smell) first noticed by the patient?

Before diagnosis, After diagnosis

7. Did the patient have any other symptoms BEFORE the development of anosmia?

Yes, No

8. What symptoms did the patient have AT THE TIME of anosmia?

None, Fever, Chills, Malaise, fatigue, Cough, Headache, Nasal Congestion, Rhinorrhea, runny nose, Gastrointestinal Distress, Other 
9. What was the condition of the COVID-19 infection at the time the anosmia was observed? Inpatient /hospitalized, Outpatient

10. Did the patient's condition worsen or improve after the anosmia was observed?

Worsen, Improve

11. What is the patient's current COVID-19 infection status?

Active, Recovered, Deceased

12. Did the anosmia resolve?

Yes, No

13. Any relevant other information

We had followed CLINICAL MANAGEMENT PROTOCOL: COVID-19, Government of India Ministry of Health and Family Welfare Directorate General of Health Services (Version 3) 13.06.20. Patients who were admitted in ward, monitored for increased work of breathing (use of accessary muscles), hemodynamic instability, increase in oxygen requirement, C-reactive protein, D-dimer and ferritin. If there is one of the above parameter is deteriorating despite standard care of management we shifted them to intensive care units. Patients who were admitted in intensive care unit were monitored for respiratory rate, Spo2, IL-6 level, D-dimer and daily chest X-ray. Patients who were admitted in ward were monitored for increased work of breathing (use of accessary muscles), hemodynamic instability and increase in oxygen requirement. We compared how many patients who had anosmia initially, and developed severe disease \& required admission and tocilizumab. We had given tocilizumab to patients who had severe pneumonia, IL-6 ten times raised, peripheral capillary oxygen saturation $(\mathrm{Spo} 2)<90 \%$ on room air and chest $\mathrm{X}$-Ray was showing more than $50 \%$ infiltrates and no associated comorbidities. Average dose of tocilizumab was $8 \mathrm{mg} / \mathrm{kg}$ of body weight subcutaneously and monitored daily with chest X-Ray finding. Patients who had neutropenia $<2000$, thrombocytopenia (platelets $<100,000$ / ul of blood, sepsis (white blood cell count not more than 10,000/uL of blood), active infections other than COVID19 and tuberculosis, impaired renal or liver function and any history of allergic reactions to monoclonal antibodies were excluded from tocilizumab therapy.

\section{Results and Data Analysis}

The Statistical Product and Service Solutions version 20 (SPSS Version 20) and CHI Square test was used to calculate the results.

In our study total number of patients were 2055 among them 1590 male and 465 were female. Average age of patients was $50.4 \pm 0.70$ years. Minimum age of COVID-
19 positive patient in our study was 18 year and maximum age was 92 year. 193 patients isolated at home with minor illness and 1862 were admitted in COVID-19 Ward. 464 patients required intensive care support among them 406 needed mechanical support. Patients who were isolated at home, $160(82.90 \%)$ had reported anosmia, among patients in COVID-19 ward, (711) $38.18 \%$ had complained about anosmia. Patients who required intensive care, but no need of mechanical ventilation communicated with us, and among 58 only 13 reported about anosmia. 406 patients needed mechanical ventilation and communication with them was not possible (Table 1) $p$ value $<0.001$ which is highly significant and showing that anosmia is inversely related to the severity of the disease. If a COVID positive patient having anosmia there is high chances, that he/she will go through mild phase of disease. Total 53 patients had received tocilizumab among them only 5 had reported anosmia out of them 4 (80\%) discharged and 1 (20\%) died. 48 Patients who received tocilizumab had no history of anosmia, among them $30(62.5 \%)$ were died \&18 (37.5\%) were discharged (Table 2). Table 2 clearly indicates that anosmia is related to good outcome even when patient goes through severe form of disease ( $p$ value 0.174 not significant due to small size of sample). Toclizumab has not significant role in decreasing mortality in severe form of disease [31 $(58.5 \%)$ out of 53 were died].

\section{Discussion}

Nasal cavity is a vital area susceptible to SARS-CoV-2 infection. The study using rhesus macaques model of coronavirus infection revealed coronaviruses have different pathogenic sites: SARS-CoV-2 (nose and throat); SARSCoV (lung); MERS-CoV (type II pneumocytes) [17].

Over last few months, an increasing number of otolaryngologists reported sudden anosmia or hyposmia may be the only presenting symptom of COVID-19 infection. In these patients, no other symptoms often seen and the diagnosis of COVID-19 could be missed. So this study was conducted to know the relationship between anosmia and severity of COVID-19 infection. Based on the telephonic conversation with COVID-19 patients who were isolated at home about their loss of smell and data collected from the COVID ward and intensive care unit (ICU) our results implicates that anosmia is inversely related with severity of disease. Among home isolated patients $82.90 \%$ reported anosmia with mild form of disease. Patients who were admitted in COVID ward with mild to moderate form of disease $38.18 \%$ complained about anosmia and among ICU patients only $22.41 \%$ reported anosmia and didn't need mechanical ventilation. No records about anosmia of mechanically ventilated patients available. Our study also 
Table 1 Prevalence of anosmia in COVID-19 patients who were isolated at home, admitted in ward and intensive care unit. Anosmia is inversely related with severity of COVID-19 disease

\begin{tabular}{|c|c|c|c|c|c|c|c|}
\hline \multirow[t]{3}{*}{ Anosmia } & \multirow{2}{*}{\multicolumn{2}{|c|}{ Home isolation }} & \multirow{2}{*}{\multicolumn{2}{|c|}{ COVID ward }} & \multicolumn{3}{|c|}{ Intensive care unit(ICU) (464) } \\
\hline & & & & & \multicolumn{2}{|c|}{ No. mechanical ventilation } & \multirow[t]{2}{*}{ Mechanical ventilation (406) } \\
\hline & No & $\%$ & No & $\%$ & No. & $\%$ & \\
\hline Present & 160 & 82.90 & 711 & 38.18 & 13 & 22.41 & No record available \\
\hline Absent & 33 & 17.10 & 1151 & 61.82 & 45 & 77.59 & \\
\hline Total & 193 & 100 & 1862 & 100 & 58 & 100 & \\
\hline
\end{tabular}

CHI square test $p$ value $<0.001$ suggestive of highly significant

Table 2 Prevalence of anosmia and outcome (in terms of death or discharge) in patients who received tocilizumab (total 53 patients received tocilizumab among them only 5 had anosmia out of them 4 discharged and 1 died). This table again proves that anosmia is related with good outcome. $p$ value- 0.174 is not significant due to small size of sample

\begin{tabular}{llllll}
\hline Outcome & Anosmia present & & \multicolumn{2}{c}{ Anosmia absent } \\
\cline { 2 - 3 } \cline { 5 - 6 } & Number & $\%$ & Number & 18 & 37.5 \\
\hline Discharge & 4 & 80 & 30 & 62.5 \\
Death & 1 & 20 & 48 & 100 \\
Total & 5 & 100 & 48
\end{tabular}

CHI square test $p$ value $=0.174$ non significant

proved that tocilzumab has no significant role in decreasing mortality $(58.49 \%$ died) in severe form of disease and there was no history of anosmia in most of patients $(90.57 \%)$ who received tocilizumab, thus anosmia is related to mild to moderate form of disease.

Mao et al. retrospectively studied the neurologic symptoms of 214 patients in Wuhan, China, and reported that $5.1 \%(\mathrm{n}=11)$ patients had anosmia [18]. They didn't report any kind of relation with severity of the disease. Katharine J. Foster et al. [19] studied that anosmia is an independent positive prognostic factor of less severe COVID-19 infection. Their study proved that anosmia was related with decreased hospitalization, ICU admission, and mechanical ventilation rates compared with the lack of anosmia. Our study supports these findings indirectly as there is less incidence of anosmia in severe form of COVID-19 infection. Lot of literature available on anosmia in COVID-19 patients, but there is deficiency when we want to know relation of anosmia with severity of disease. Our study is helpful to give an idea to the otolaryngologist and general physician that if patient is having anosmia subsequently he/she will go through mild to moderate phase of disease. A study by Samaee H et al. [20] recommended that tocilizumab have positive effect in improvement of damage of lung and arterial oxygen saturation. In our study we didn't find any effect of tocilizumab in COVID-19 pneumonia.

A retrospective cohort study by Guaraldi G. et al. [21] proposed that tocilizumab in COVID-19 patients whether administered intravenously or subcutaneously, might reduce the risk of invasive mechanical ventilation or death in patients with severe COVID-19 pneumonia.

Another study by Vu, C.A. et al. [22] effects of tocilizumab in COVID-19 patients: a cohort study recommended that majority of patients shown clinical improvement and were successfully discharged alive from the hospital after receiving tocilizumab. They revealed that, tocilizumab may have a role in the early treatment of COVID-19. However larger randomized controlled studies are needed to confirm this. Findings of our study are contrary to above findings. Main limitation of our study was, we didn't compare outcome (death and discharge) in patients who received tocilizumab \& who didn't received. There was less number of anosmia in patients who received tocilizumab, so due to inadequate sample size $p$-value was not significant (Table 2) but this indicates that requirement of tocilizumab is lower in patients who had anosmia as compare to those who don't have anosmia. Another limitation of our study was, that anosmia reported by patients only on telephonic conversation which was a subjective finding and we didn't 
perform any kind of olfactory function test in these patients.

\section{Conclusion}

COVID-19 virus is most likely associated with divergent mutations and clinical form, and disease is new as of yet, there remain more questions than answers. COVID-19 infection is associated with chemosensitive dysfunction and anosmia may be the only symptom that presents the disease. Due to anosmia, first contact of patient is with otorhinolaryngologist. When patient presents with only anosmia to the otorhinolaryngology department, COVID test should get done first and if test is positive, isolate the patient at home. Tocilizumab may be given in severe form of disease as per protocol but has no significant role in decreasing mortality.

Author Contributions First draft of the manuscript was written by Manju Silu and all authors commented on previous versions of the manuscript. All authors read and approved the final manuscript.

Data Availability Data of this study is original and not copied from any other source available. This is an original research done in a premier government institute. Data of this study has not been submitted anywhere else in the past for the purpose of publication. The data used to support the findings of this study are included within the article.

\section{Declarations}

Conflict of interest The authors declare that there is no conflict of interest regarding the publication of this article.

Ethical Approval Statement Here we are declaring that laws which are applicable to our article are followed. Ethical committee approval was taken before commencing this study.

\section{References}

1. Zhou F, Yu T, Du R et al (2020) Clinical course and risk factors for mortality of adult inpatients with COVID-19 in Wuhan, China: a retrospective cohort study. Lancet 395:1054-1062

2. Guan W-J, Ni Z-Y, Hu Y et al (2020) Clinical characteristics of coronavirus disease 2019 in China. N Engl J Med 382:1708-1720

3. Nicastri E, D'Abramo A, Faggioni G et al (2020) Coronavirus disease (COVID-19) in a paucisymptomatic patient: epidemiological and clinical challenge in settings with limited community transmission Italy. Euro Surveill 25(11):2000230

4. Lechien JR, Chiesa-Estomba CM, De Siati DR, Horoi M et al (2020) Olfactory and gustatory dysfunctions as a clinical presentation of mild-to-moderate forms of the coronavirus disease (COVID-19): a multicenter European study. Eur Arch Otorhinolaryngol 277:2251-2261
5. Eliezer M, Hautefort C, Hamel AL, Verillaud B, Herman P, Houdart E, Eloit C (2020) Sudden and complete olfactory loss function as a possible symptom of COVID-19. JAMA Otolaryngol Head Neck Surg 146:674

6. Lechien JR, Chiesa-Estomba CM, Place S, Van Laethem Y et al (2020) Clinical and epidemiological characteristics of 1420 European patients with mild-to-moderate coronavirus disease 2019. J Intern Med 288(3):335-344

7. Parma V et al (2020) More than smell COVID-19 is associated with severe impairment of smell, taste, and chemesthesis. Chem Senses 45(7):609-622

8. Desforges M, Le Coupanec A, Brison E et al (2014) Neuroinvasive and neurotropic human respiratory coronaviruses: potential neurovirulent agents in humans. Adv Exp Med Biol 807:75-96

9. Li Y, Bai W, Hashikawa T (2020) The neuroinvasive potential of SARS-CoV2 may play a role in the respiratory failure of COVID19 patients. J Med Virol 92(6):552-555

10. Suzuki M, Saito K, Min WP et al (2007) Identification of viruses in patients with postviral olfactory dysfunction. Laryngoscope 117:272-277

11. Giacomelli A, Pezzati L, Conti F et al (2020) Self-reported olfactory and taste disorders in SARS-CoV-2 patients: a crosssectional study. Clin Infect Dis 71(15):889-890

12. Siddiqi HK, Mehra MR (2020) COVID-19 illness in native and immunosuppressed states: a clinical-therapeutic staging proposal. J Heart Lung Transplant 39:405-407

13. Huang C, Wang Y, Li X et al (2020) Clinical features of patients infected with 2019 novel coronavirus in Wuhan, China. Lancet 395:497-506

14. Pedersen SF, Ho Y-C (2020) SARS-CoV-2: a storm is raging. J Clin Invest 130:2202-2205

15. Hennigan S, Kavanaugh A (2008) Interleukin-6 inhibitors in the treatment of rheumatoid arthritis. Ther Clin Risk Manag 4:767-775

16. Swerdlow DI, Holmes MV, Kuchenbaecker KB et al (2012) The interleukin-6 receptor as a target for prevention of coronary heart disease: a mendelian randomisation analysis. Lancet 379:1214-1224

17. Rockx B, Kuiken T, Herfst S, Bestebroer T, Lamers MM, Oude Munnink BB (2020) Comparative pathogenesis of COVID-19, MERS, and SARS in a nonhuman primate model. Science 368:1012-1015

18. Mao L, Jin H, Wang M, Hu Y, Chen S, He Q (2020) Neurologic manifestations of hospitalized patients with coronavirus disease 2019 in Wuhan. China JAMA Neurol 77(6):683-690

19. Foster KJ, Jauregui E, Bobby Tajudeen M, Bishehsari F, Mahdavinia M (2020) Smell loss is a prognostic factor for lower severity of coronavirus disease 2019. Ann Allergy Asthma Immunol 125:475-494

20. Samaee H, Mohsenzadegan M, Ala S, Maroufi SS, Moradimajd P (2020) Tocilizumab for treatment patients with COVID-19: recommended medication for novel disease. Int Immunopharmacol 89(Pt A):107018. https://doi.org/10.1016/j.intimp.2020.107018

21. Guaraldi $G$ et al (2020) Tocilizumab in patients with severe COVID-19: a retrospective cohort study. Lancet Rheumatol 2(8):E474-E484

22. Vu CA, DeRonde KJ, Vega AD et al (2020) Effects of tocilizumab in COVID-19 patients: a cohort study. BMC Infect Dis 20:964. https://doi.org/10.1186/s12879-020-05701-4

Publisher's Note Springer Nature remains neutral with regard to jurisdictional claims in published maps and institutional affiliations. 